\title{
Ideas de los estudiantes universitarios sobre las relaciones trabajo y energía en Mecánica en cursos introductorios de Física
}

University students' ideas on the relations between work and energy in Mechanics in introductory Physics courses

\author{
Jose Gutierrez-Berraondo $^{1}$, Kristina Zuza ${ }^{2}$, Genaro Zavala ${ }^{3,4}$, Jenaro Guisasola*5 \\ ${ }^{1}$ Instituto de Maquina Herramienta IMH Elgoibar, Elgoibar, Spain \\ ${ }^{2}$ Universidad del País Vasco, Escuela de Ingeniería de Gipuzkoa San Sebastian, Spain \\ ${ }^{3}$ Tecnologico de Monterrey, Escuela de Ingenieria y Ciencias, Monterrey, Mexico \\ ${ }^{4}$ Universidad Andres Bello, Facultad de Ingenieria, Santiago, Chile \\ ${ }^{5}$ Universidad del Pais Vasco, Escuela Universitaria Politecnica de San Sebastian, Plaza Europa 1, Donostia, Spain
}

Recibida en 17 de Abril, 2017. Revisado en 17 de Junio, 2017. Aceptado en 7 de Julio, 2017.

\begin{abstract}
Las relaciones entre trabajo y energía continúan siendo fuentes de problemas de enseñanza/aprendizaje en los cursos introductorios de física a nivel universitario, ya que implican conceptos abstractos y habilidades de metodología científica. Teniendo en cuenta los estudios anteriores en la literatura, el objetivo principal de esta contribución es identificar y documentar las dificultades específicas que los estudiantes muestran al pensar y razonar sobre la aplicación del concepto de trabajo, y la relación entre trabajo y energía en sistemas aislados. Esta investigación consiste en el análisis de respuestas a un cuestionario trabajo-energía administrado a estudiantes de los cursos introductorios de física de la Universidad del País Vasco. Se presentan los resultados de cuatro preguntas planteadas a los alumnos donde se demuestra que, después de la instrucción, los estudiantes siguen teniendo problemas en calcular el producto escalar en la definición de trabajo y que una buena parte de estudiantes tiene dificultades en establecer relaciones correctas entre el trabajo realizado por una fuerza externa al sistema y la variación de energía del sistema. Al final se establecen las conclusiones generales del trabajo donde se hacen recomendaciones al instructor e investigador.
\end{abstract}

Palabras clave: Investigación en enseñanza de la física en universidad, Principio Generalizado del Trabajo y la Energía, trabajo, aprendizaje de los estudiantes.

Relations between work and energy are still a source of teaching-learning problems in the first years of university introductory physics courses since they involve abstract concepts and skills of scientific methodology. Considering the previous studies in the literature, the main objective of this contribution is to identify and document specific difficulties that students show while thinking and reasoning about the relation between work and energy in isolated systems. This research consists in the analysis of answers to a work-energy questionnaire administered to students taking introductory physics courses at the University of the Basque Country (Spain). The results of four questions presented to the students show that, after the instruction, students still have problems calculating the scalar product in the definition of work, and that a good portion of students have difficulties in establishing correct relations between the work done by an external force to the system and the energy variation of the system. At the end, general conclusions of this contribution are established where recommendations are made to the instructor and researcher.

Keywords: physics education research at university, General Principle on work and energy, work, students' learning.

\section{Introducción}

En las últimas décadas un número creciente de investigaciones muestran que la enseñanza y el aprendizaje de los conceptos de trabajo, energía y las relaciones entre ellos, son particularmente complejos y, que existe una preocupación sobre el tema entre profesores e investigadores

*Correo electrónico: jenaro.guisasola@ehu.es.
[1]. La mayoría de las investigaciones se han desarrollado en los niveles pre-universitarios [2, 3], y pocos estudios se han llevado a cabo en el nivel universitario $[4,5]$. El trabajo que presentamos se sitúa en cursos universitarios de física general para estudiantes de primer curso de ciencias e ingeniería.

La introducción del concepto de trabajo en libros de texto estándar implica una discusión de una fuerza $\mathrm{F}$ 
aplicada a un objeto que se desplaza $\Delta$ r. Esta explicación, continúa con la presentación de la siguiente ecuación o una variación de la misma: $W=\vec{F} \cdot \Delta \vec{r}=\mathrm{F} \Delta \mathrm{r} \cos \theta$. Sin embargo, muchos libros de texto usan declaraciones generales como " $\Delta \mathrm{r}$ es el desplazamiento del objeto" o simplemente "el desplazamiento", sin identificar lo que está siendo desplazado. En relación con el término "fuerza", a menudo ocurre que hay múltiples fuerzas que actúan sobre el sistema. En estos casos es necesario discutir cómo calcular el trabajo neto de todas las fuerzas [6]. A menudo, los profesores y los libros de texto se centran en discutir los efectos de las fuerzas en el objeto, pero cuando la situación es más compleja y el objeto es deformable, es más beneficioso pensar en "sistemas" que en "objetos" [4]. Como resultado, es necesario definir la relación entre trabajo y energía introduciendo magnitudes como "pseudo-trabajo" [7]. Durante un desplazamiento, si consideramos una fuerza que actúa sobre el centro de masa de un objeto, el trabajo resultante realizado por la fuerza es la magnitud llamada "pseudo-trabajo". Estas imprecisiones pueden conducir a dificultades conceptuales cuando los estudiantes aplican las definiciones en diferentes contextos como objetos sobre los que actúan fuerzas de fricción o fuerzas aplicadas a objetos deformables o bien, objetos girando $[8,9]$. Por tanto, aunque el "trabajo" es una palabra común en el lenguaje cotidiano, con muchos significados, la física utiliza "trabajo" en el sentido de energía transferida 'a' o 'desde' un sistema por la aplicación de la fuerza. En física, la cantidad de trabajo mide la energía transferida hacia un sistema o desde un sistema, por una fuerza externa. El cálculo de la energía transferida por la fuerza es $W=\int_{a}^{b} \vec{F} \cdot \overrightarrow{d r}$.

La misma rigurosidad que se utiliza para la definición de trabajo, se aplica en física para definir la relación entre trabajo y energía que implica diferentes tipos de energía, no sólo la energía cinética $[1,3]$. Frecuentemente, la falta de definición explícita del "sistema" a analizar y los diferentes tipos de energía involucrados, suele conducir a dificultades conceptuales cuando los estudiantes aplican la relación entre trabajo y energía en diferentes contextos [10]. La falta de concreción en el análisis podría ser un problema en casos simples como los sistemas de una sola partícula y podría ser más problemático en el estudio de los sistemas mecánicos en los que el estudiante encuentra trabajos realizados por fuerzas de fricción o por fuerzas aplicadas a objetos deformables (muelles)

Teniendo en cuenta las investigaciones anteriores, el objetivo principal de este estudio es identificar y documentar las dificultades específicas que los alumnos presentan en la comprensión del concepto de trabajo y de su relación con la variación de energía en sistemas de partículas. Nuestras preguntas de investigación son las siguientes:

1. ¿Cuáles son las dificultades que tienen los estudiantes universitarios al aplicar el concepto de trabajo?

2. ¿Qué dificultades de comprensión tienen los estudiantes cuando relacionan el trabajo y los cambios de energía al aplicar el Principio Generalizado del Trabajo y la Energía en mecánica?

Nuestro estudio aborda la descripción de las ideas de los estudiantes sobre el concepto de trabajo y sus relaciones con el cambio de energía en un sistema de partículas. En particular, analizaremos cómo utilizan el concepto de trabajo y la relación con los cambios de energía, al abordar diferentes situaciones en el contexto de la Mecánica. Este estudio forma parte de un proyecto de investigación más amplio cuyo objetivo es diseñar, implementar y evaluar una secuencia de enseñanza-aprendizaje sobre el Principio Generalizado del Trabajo y la Energía (PGTE) para cursos de nivel básico de física en la universidad.

El trabajo presentado aquí añade a la investigación previa sobre dificultades de los estudiantes al aplicar el concepto de trabajo y sus relaciones con los cambios de energía, que se centra en el nivel de enseñanza universitaria. Este es un aspecto, hasta donde conocemos, muy poco analizado por la investigación en Enseñanza de la Física. Además, el estudio también se centra en un aspecto poco investigado, al analizar las relaciones entre trabajo realizado en el sistema y la correspondiente variación de energía, utilizando el PGTE para un sistema de partículas. Nuestros resultados pueden ser aprovechados por profesores, escritores de libros de texto y diseñadores de planes de estudios al planificar actividades más significativas en el capítulo del trabajo y el PGTE en mecánica.

Este articulo, en primer lugar describirá la metodología utilizada para responder a las preguntas de investigación y el contexto en el que se ha llevado a cabo. A continuación, se mostrarán los resultados obtenidos. Para finalizar se hará una discusión de los resultados y sus implicaciones para la enseñanza de la física.

\section{Diseño y Metodología}

Para responder a las preguntas de investigación, desarrollamos un cuestionario con cuatro cuestiones abiertas centradas en la explicación. Las preguntas presentan contextos sencillos desde el punto de vista matemático, de forma que las dificultades de desarrollo matemático no enmascaren las dificultades de comprensión. El objetivo principal de esta contribución es entender el nivel de comprensión de los estudiantes de grado sobre las ideas básicas que intervienen en la definición del trabajo y el PGTE en mecánica. Por lo tanto, nuestros alumnos de la Escuela de Ingeniería de Gipuzkoa y de la Escuela IMHElgoibar de la Universidad del País Vasco (UPV/EHU) asistieron a clases para profundizar en estos conceptos antes de contestar el cuestionario. Todos los estudiantes de primer año de la UPV / EHU habían recibido dos años de física en la escuela secundaria y estaban haciendo su primer curso de física para estudiantes de ingeniería. Los estudiantes recibieron 3,5 horas de clases por semana y 2 horas de laboratorio por mes durante 15 semanas 
(primer semestre) en Mecánica. Las clases magistrales fueron impartidas por instructores experimentados del Departamento de Física. Dentro del curso se dedican dos o tres semanas a enseñar el capítulo del trabajo, la energía y el PGTE. El objetivo de las clases magistrales y las clases de resolución de problemas es enseñar los conceptos de energía cinética, energía potencial, fricción cinética, conservación de energía mecánica, masa y energía y los cálculos de energía. También se analiza en detalle cómo se aplica el principio de conservación de la energía en diferentes situaciones con fricción.

Para validar las preguntas del cuestionario, se realizó un estudio piloto con 30 estudiantes de primer año. El estudio piloto confirmó que los estudiantes no tenían problemas para entender cómo se formularon las preguntas. Además, los objetivos de cada pregunta fueron validados por tres profesores de primer año de ingeniería que estuvieron de acuerdo con los contenidos. Se realizó una nueva versión del cuestionario, en la que se recogían sugerencias de redacción, pero sin variar el objetivo principal de las mismas. Esta versión es la que se pasó a 148 estudiantes. Las preguntas se incluyeron en los exámenes de primer año que formaban parte del sistema de evaluación Dado que los resultados de las dos escuelas de la Universidad del País Vasco no difieren significativamente, se han agrupado en los resultados presentados en este artículo.

Con el objetivo de caracterizar las respuestas, se codificaron los comentarios reconocidos como "una explicación", basados en categorías con rasgos fácilmente reconocibles, tales como declaraciones científicas y argumentación [11]. Esto implicó que un miembro del equipo de investigación leyera las respuestas de los estudiantes y derivara un borrador de categorías de descripción para cada pregunta. A continuación otros dos investigadores analizaron las respuestas de los estudiantes y asignaron tentativamente cada respuesta a una de las categorías del borrador. Una vez clasificadas las respuestas, se compararon las asignaciones de respuesta y se alcanzó un grado de acuerdo muy significativo con un coeficiente de confiabilidad de la kappa de Cohen de 0,86. Cualquier desacuerdo sobre la descripción de la categoría o asignación de respuestas se resolvió al referirse a las respuestas como la única evidencia de la comprensión de los estudiantes. Se utilizó un proceso iterativo para definir las categorías finales [12].

A continuación, describimos los objetivos de las cuatro preguntas del cuestionario (ver anexo). La cuestión Q1 indaga sobre la aplicación del concepto de trabajo en una situación sencilla donde la dirección de la fuerza aplicada y la dirección del movimiento del sistema son la misma. Los estudiantes tienen que aplicar la definición de trabajo realizado por la fuerza externa sobre el sistema considerando que el producto escalar de dos vectores. En las cuestiones Q2 y Q3 se repite el objetivo de indagar si los estudiantes tienen dificultades en aplicar la definición de trabajo, pero en estas situaciones deben considerar el ángulo formado por la dirección de la fuerza y la dirección del movimiento del sistema. Además, en las cuestiones Q1, Q2 y Q3, se solicita a los estudiantes que indiquen la relación entre el trabajo realizado por una fuerza externa al sistema y la variación de energía en el mismo. Así pues, los estudiantes deben aplicar el PGTE para situaciones simples donde sólo hay una fuerza externa y variación de energía cinética ya que se han simplificado los demás tipos de energía (no hay rozamiento, el sistema se considera como una partícula con toda la masa situada en el centro de masas).

En la cuestión Q4 se pregunta a los estudiantes sobre un caso particular del PGTE, como es el teorema de la energía cinética. Los estudiantes deben identificar las condiciones que se tienen que cumplir para aplicar el teorema de la energía cinética. Deben darse cuenta que según las condiciones de contorno el PGTE adopta una forma diferente. Por ejemplo, los estudiantes razonan adecuadamente cuando indican que en los sistemas formados por una sola partícula el PGTE se reduce a la expresión del Teorema de la Energía Cinética, o bien, cuando reducen las condiciones de forma que sólo haya energía cinética y trabajo externo. En la cuestión se solicita a los estudiantes que justifiquen su respuesta explicando un ejemplo, de esta forma podemos analizar con mayor detalle los argumentos de los estudiantes.

\section{Resultados y Discusión}

En esta sección mostramos los resultados obtenidos en ambos grupos de estudiantes para las preguntas descritas anteriormente. En las discusiones identificaremos algunas dificultades conceptuales, que parecen ser comunes en muchos estudiantes. La descripción de las ideas de los estudiantes se concentrará en algunas dificultades persistentes y específicas.

En la tabla 1 se resumen las categorías de las respuestas de los estudiantes en relación a las cuestiones Q1, Q2 y Q3.

Las tres cuestiones presentan diferentes contextos pero los porcentajes de respuestas en cada categoría son similares, lo que indica la consistencia de las respuestas de los estudiantes ante cuestiones con el mismo objetivo aunque en diferentes contextos.

Hemos identificado tres categorías comunes de razonamiento que surgieron cuando los estudiantes respondieron a cada pregunta. Hay una categoría en cada pregunta que incluye todos los elementos que corresponden a un experto en la aplicación del concepto de trabajo (categoría A, sobre el $40 \%$ ) "Calcula el trabajo correctamente". Las explicaciones en esta categoría, calculan correctamente el producto escalar de la definición de trabajo realizado por la fuerza externa. La mayoría de estas respuestas en la cuestión Q1 $(35,5 \%)$, la mitad de las respuestas en la cuestión Q2 (20,5\%), y una minoría de las respuestas en la cuestión Q3 (15,0\%), establecen correctamente la relación entre el trabajo realizado y la variación de energía 
Tabla 1: categorías de respuestas de los estudiantes a las cuestiones Q1, Q2 y Q3.

\begin{tabular}{|c|c|c|c|}
\hline \multirow[t]{2}{*}{ Categorías } & \multicolumn{3}{|c|}{ Estudiantes $(\mathrm{N}=148)$ Porcentaje de respuestas } \\
\hline & Cuestión Q1 & Cuestión Q2 & Cuestión Q3 \\
\hline A - Calcula el trabajo correctamente: & 40,5 & 41,5 & 40.5 \\
\hline A.1. Lo relaciona con la variación de la energía correctamente & 35,5 & 20,5 & 15,0 \\
\hline A.2. No relaciona el trabajo y la energía & 5,0 & 21,0 & 25.5 \\
\hline B- Tiene dificultades al calcular el trabajo y afirman que la energía varía: & 21,0 & 17,5 & 23,0 \\
\hline B.1. Dificultades en el producto escalar & 0.0 & 2,5 & 6,0 \\
\hline B.2. No tienen en cuenta el producto escalar y consideran la fuerza como un escalar & 21,0 & 15 & 17,0 \\
\hline C.1. Elementos aislados del marco teórico energético & 0,0 & 2,0 & 1,5 \\
\hline D- Incoherente. & 19,5 & 13,0 & 10,0 \\
\hline NC- No contesta & 19,0 & 19,0 & 20,0 \\
\hline
\end{tabular}

del sistema (categoría A.1.). Ejemplos de este tipo de respuestas son:

"El trabajo realizado es $W=F \cdot r \cdot \cos \alpha=6$ Julios . El trabajo realizado sobre la pastilla de Hockey aumenta su energía, en su desplazamiento no hay incremento de la energía potencial, por lo tanto, habrá que ver cómo varía su energía cinética. Aplicando el Principio de la Energía $W_{T}=\Delta K$ por no haber fuerza de rozamiento, se produce aumento de Energía Cinética en 6 Julios" (Cuestión Q1, estudiante 38)

"El trabajo realizado será $W=3 \cdot 2 \cdot \cos 90^{\circ}$ $=0$ Julios. El trabajo se transforma en la energía cinética de manera que $W=\Delta K=0$ " (Cuestión Q2, estudiante 110)

"El trabajo realizado en relación a la energía significa que el trabajo se puede transformar en energía, ya que la energía es la capacidad de hacer un trabajo. El trabajo realizado es $W=3 \cdot 2 \cdot \cos 150^{\circ}=-5,196$ Julios " (Cuestión Q3, estudiante 79)

Hay otras explicaciones que calculan correctamente el trabajo pero no mencionan su relación con la variación de energía o bien, realizan afirmaciones sobre la variación de energía que no justifican (categoría A.2). Por ejemplo:

"El trabajo realizado es $W=3 \cdot 2 \cdot \cos 90^{\circ}=$ 0 Julios"(Cuestión Q2, estudiante 95)

"El trabajo realizado disminuye la energía del sistema" (Cuestión Q3, estudiante 90)

En la categoría A.2 se encuentran los estudiantes que son capaces de entender las relaciones matemáticas del concepto de trabajo (en sus diferentes versiones de acuerdo a cada cuestión), es decir, que las cantidades involucradas son la fuerza externa sobre el sistema y su desplazamiento, además de que estas magnitudes son vectoriales y por último la operación matemática. Sin embargo, no son capaces de relacionar este resultado con la energía del sistema. Al parecer, estos estudiantes se quedan en la parte operativa del problema sin llegar a entender conceptualmente que el trabajo hecho por esta fuerza externa tiene una consecuencia sobre el sistema.

En la categoría B "Tiene dificultades al calcular el trabajo y afirman que la energía varía", se incluyen explicaciones que calculan incorrectamente el trabajo realizado y hay dificultades en: 1) Aplicar el producto escalar considerando ángulos que no son correctos (categoría B.1); 2) no se tiene en cuenta el carácter vectorial de las magnitudes incluidas en el cálculo del trabajo (categoría B.2). La relación del trabajo con la variación de energía se realiza de forma general (aumenta o disminuye) y frecuentemente, de forma incorrecta debido al cálculo erróneo del trabajo realizado. Por ejemplo:

"El trabajo realizado es $W=F \cdot 2 \cdot \cos 30^{\circ}=$ $3 \cdot(2) \cdot \cos 30^{\circ}=5,19$ Julios. La energía de la caja varía" (Cuestión Q3, estudiante 77)

"El trabajo realizado es $W=F \cdot \Delta r=3 \cdot 2$ $=6 \mathrm{~N}$. La energía de la caja no varía ya que la velocidad es la misma" (Cuestión Q2, estudiante 5)

En esta categoría están los estudiantes que se quedan en la parte operativa del cálculo del trabajo, pueden observar que están involucradas las cantidades fuerza y desplazamiento, pero no entienden las relaciones correctas para encontrar el producto escalar necesario. Además, tienen dificultades conceptuales, desde no poder argumentar el cambio de energía cinética en función del resultado del trabajo hasta no saber conectarlo con este resultado.

En la categoría C, se incluyen respuestas minoritarias (menos del 10,0\%) que presentan elementos aislados o mal memorizados de la teoría del trabajo y la energía (categoría C.1) o de la teoría dinámica (categoría C.2). Este tipo de explicaciones no contestan a la pregunta. Por ejemplo:

\section{"Siempre que hay trabajo varía la energía" (cuestión Q3, estudiante87)}

En la tabla 2 se resumen las categorías de las respuestas de los estudiantes a la cuestión Q4. En la cuestión se pidió a los estudiantes que explicaran la validez del teorema de energía cinética y sus límites (ver sección anterior). Tres categorías mutuamente excluyentes se dedujeron de las 
Tabla 2: categorías de respuesta a la cuestión Q4

\begin{tabular}{lc}
\hline Categorías & Estudiantes (N=148) Porcentaje de respuestas \\
\hline A- Reconoce el campo de validez del Teorema & 29.5 \\
A.1. Relaciona correctamente W y $\Delta$ E. & 3,0 \\
A.2. Afirmaciones generales en contra de la generalidad del teorema. & 26.5 \\
B- Argumentos incorrectos en contra de la generalidad del teorema. & 5.5 \\
C.1. De acuerdo con la afirmación. & 17.5 \\
C.2. Argumentos incorrectos. & 7,0 \\
D- Incoherente. & 17.5 \\
NC- No contesta & 23,0 \\
\hline
\end{tabular}

respuestas de los estudiantes. Las categorías representan diferentes maneras de pensar sobre las relaciones entre el trabajo realizado y los cambios de energía. Las ideas de que "el teorema es válido en general" (categoría C) era bastante común entre los estudiantes (alrededor de un cuarto de las respuestas). Otros estudiantes responden con "declaraciones generales contra la generalidad del Teorema" (categoría A.2) o bien, proponen "argumentos incorrectos contra la generalidad del Teorema" (categoría B).

En la categoría A se incluyen las respuestas que utilizan correctamente dos argumentos diferentes: a) cuando el sistema está formado por una sola partícula el tipo de energía es cinética y por lo tanto, el teorema es válido en general (A.1); b) existen más tipos de energía que afectan las relaciones entre trabajo y energía, por lo que el teorema no es válido (A.2). Algunos ejemplos son:

"Sería válido siempre y cuando definamos el sistema de tal manera que sólo hay una partícula. Al tener una sola partícula, no habrá ningún cambio en la energía potencial, ya que no habría referencia alguna" (categoría A.1)

"No es válido en general. La energía mecánica es la suma de la energía cinética y la energía potencial. $E_{m}=E_{C}+E_{P}=1 / 2 \cdot m \cdot v^{2}+$ $m \cdot g \cdot h$. La energía cinética es la energía que tiene un cuerpo al estar en movimiento. La energía potencial es la energía que tiene un cuerpo por el mero hecho de estar a una altura". (Categoría A.2)

Es importante indicar que en la categoría A se incluyen respuestas que argumentan las restricciones del teorema de la Energía Cinética debido al sistema analizado o debido a los otros tipos diferentes de energía en los cuales el trabajo puede ser transformado.

La categoría B incluye respuestas donde los estudiantes discuten un caso particular, como un ejemplo de que el teorema no se cumple. Por ejemplo:

"Esa ecuación no siempre es válida. Por ejemplo, un ciclista que sube una colina al alcanzar la altura máxima la energía cinética será 0 y la energía cinética se transformará en potencial" (categoría B)
Los estudiantes en esta categoría saben que el teorema no es válido, pero no saben argumentar científicamente por qué es así. Normalmente sus ejemplos, aunque la mayoría de ellos aplicables, tenían concepciones que se apartan de la concepción científica del fenómeno explicado. En este tipo de respuestas la dificultad principal es de tipo conceptual al no entender que el campo de validez de las magnitudes incluidas en el teorema depende del sistema físico que se define. No hablan del sistema físico en el que se aplica el balance de energía.

Hay una cuarta parte de las respuestas que indican que el teorema de energía cinética es válido para todos los casos en Mecánica (categoría C.1) o que la validez depende del tipo de fuerza en juego (categoría C.2). Los estudiantes argumentan que el teorema es una ley general en Mecánica o que es válida si la fuerza es conservadora. Por ejemplo:
"Sí, el trabajo es siempre el cambio en la energía cinética. En una competición el cam- bio de velocidad de un corredor es trabajo y este trabajo es a menudo la variación de la energía cinética" (categoría C.1)
"No siempre es válido, porque todos los tipos de fuerzas no son iguales, unas son conserva- doras y otras no conservadoras, por ejemplo, el trabajo realizado por la gravedad" (cate- goría C.2)

Los resultados sugieren que una proporción significativa de estudiantes presentan confusión entre conceptos aislados del marco teórico (fuerza, fuerzas conservadoras y no conservadoras, trabajo y energía). Además, al analizar la validez del teorema no pueden explicar un argumento (categoría C) o lo hacen de manera inconsistente. En este tipo de respuestas se mezclan dificultades conceptuales de la comprensión de los conceptos incluidos en el teorema. Hay respuestas que presentan confusión entre los conceptos de fuerza y trabajo o entre trabajo y energía. Las respuestas incluidas en esta categoría muestran un bajo nivel de comprensión de los conceptos básicos de la mecánica que imposibilita un análisis correcto del balance de energía.

Hay un porcentaje importante de respuestas en todas las cuestiones, que no discuten lógicamente o simplemente no responden. No parece que el porcentaje de estudiantes que no responden pueda atribuirse a la falta de interés, 
ya que han respondido con interés al cuestionario y también el puntaje obtenido, contribuye para la nota final. Por tanto, este tipo de respuestas puede atribuirse a la dificultad que tienen los estudiantes para contestar a este tipo de preguntas donde tienen que utilizar argumentos con significados científicos que van más allá de aplicar mecánicamente una fórmula.

\section{Conclusiones}

Este estudio revela una rica y robusta variedad de dificultades de los estudiantes en el cálculo del trabajo realizado por una fuerza aplicada sobre un sistema y en las relaciones trabajo y energía. Las tendencias en las respuestas de los estudiantes fueron consistentes entre las diferentes situaciones analizadas e independientes del instructor de la Escuela de Ingeniería. Los cursos tradicionales de Física a nivel introductorio en la universidad tienen algo de éxito cuando enseñan el cálculo del trabajo (40\% en cuestiones Q1, Q2 y Q3, categoría A). Sin embargo este porcentaje de éxito es significativamente menor al analizar las relaciones trabajo y energía, según el contexto analizado del (35,5\%-15\% en cuestiones Q1, Q2 y Q3, categoría A.1). Además, solo un tercio de los estudiantes reconoce los límites de aplicabilidad del Teorema de la Energía Cinética (cuestión Q4, categoría A). El estudio muestra evidencias de que las dificultades en el cálculo del concepto de trabajo y sus relaciones con la variación de energía siguen persistiendo después de la instrucción.

En este estudio hemos planteado dos preguntas de investigación: 1) ¿Cuáles son las dificultades que tienen los estudiantes universitarios al aplicar el concepto de trabajo? y 2) ¿Qué dificultades de comprensión tienen los estudiantes cuando relacionan el trabajo y los cambios de energía al aplicar el Principio Generalizado del Trabajo y la Energía en mecánica? Abordaremos estas dos preguntas con los hallazgos en el estudio.

En relación a la primer pregunta de investigación, una fracción significativa de estudiantes (alrededor del $20 \%$ en cuestiones Q1, Q2 y Q3, categoría B), después de la instrucción, siguen teniendo problemas en calcular el producto escalar en la definición de trabajo. Esta dificultad se mantiene $(17,5 \%$ categoría B) incluso en la cuestión Q2 que muestra el caso extremo de una fuerza perpendicular a la dirección del movimiento. Nuestros resultados sugieren que los estudiantes no consideran como vectores las magnitudes del producto escalar incluido en la definición de trabajo, y por tanto evitan hablar del ángulo formado por la dirección de la fuerza y la dirección del movimiento. Parece que los estudiantes consideran que como la magnitud trabajo es un escalar, no se deben considerar los vectores incluidos en el producto escalar de la definición operativa de trabajo. En los contextos más complejos donde la cuestión indica el ángulo formado por el vector fuerza y la dirección del desplazamiento (cuestión Q3) los estudiantes no consideran el ángulo correcto entre los vectores. Estos resultados son convergentes con otros estudios internacionales en países diferentes que muestran que los estudiantes tienen dificultades en el cálculo del producto escalar tanto en contextos de Mecánica [13] como de Electromagnetismo [14]

En relación a la segunda pregunta de investigación podemos observar que, a pesar de la instrucción, una fracción importante de estudiantes tiene dificultades en establecer relaciones correctas entre el trabajo realizado por una fuerza externa al sistema y la variación de energía producida en dicho sistema. Sólo un $30 \%$ de los estudiantes (categoría A, cuestión Q4) ha desarrollado un marco coherente del uso del teorema trabajo-energía cinética. Alrededor de la cuarta parte de estudiantes (categoría C, cuestión Q4) creen que el teorema es válido en cualquier situación sin tener en cuenta los diferentes tipos de energía que podrían estar involucrados en el sistema analizado. En consecuencia, los estudiantes no son capaces de definir qué tipo de energía tiene el sistema, ni de identificar las fuerzas externas al mismo. Por otro lado, hay estudiantes que han aprendido que el teorema no es válido; sin embargo, no han entendido por qué. Los ejemplos dados por estos estudiantes demuestran que su marco conceptual que implica trabajo, energía o incluso fricción no es adecuado.

Antes de extraer conclusiones e implicaciones para la enseñanza, hay que tener en cuenta que el cuestionario fue realizado en dos escuelas de ingeniería de la Universidad País Vasco. Por lo tanto, no podemos asegurar la evidencia de contextos más generales. Sin embargo, hemos comprobado que los resultados obtenidos en este estudio coinciden con los resultados encontrados en otros estudios realizados en otros países.

Los resultados obtenidos presentan nuevas características del pensamiento de los estudiantes, lo que demuestra que un porcentaje significativo de ellos no puede interpretar relaciones trabajo y energía en contextos sencillos, donde no es necesaria la utilización de instrumentos matemáticos complejos. Por otra parte, los resultados de este estudio también muestran que es necesario enfatizar el significado de cada concepto que participa en el PTGE en Mecánica de cursos introductorios. Sería necesario, en primer lugar, definir el sistema y las fuerzas que actúan sobre él así como analizar la trayectoria descrita por el sistema. Puede parecer simple este primer punto, sin embargo, el estudiante tiene grandes dificultades en cada uno de las acciones que se presentan. La definición del sistema y las fuerzas que están involucradas sobre este sistema van unidas ya que el estudiante tiene que entender que hay fuerzas internas y externas y que su clasificación depende de la definición del sistema. Además, sería importante, en este momento, enfatizar sobre los pares de fuerza de la tercera Ley de Newton el cual, de acuerdo a la literatura, se mantiene como un problema, no sólo a través del curso de mecánica, sino también posterior a llevarlo. En segundo lugar, enfatizar sobre las características vectoriales de los términos involucrados 
en el cálculo del trabajo y las consecuencias que esto tiene en la operación que se tiene que hacer. Es decir, el estudiante debe entender que tanto la fuerza sobre el sistema como su desplazamiento son cantidades que, además de tener magnitud, tienen dirección y que esto tiene consecuencias. En la enseñanza de la mecánica, no estaría de más hacer una pausa para enfatizar en las relaciones matemáticas del producto escalar de una manera activa. Una opción en utilizar actividades tutoriales como los que han sido propuestos [15]. Por último, analizar los diferentes tipos de energía involucrados para que los estudiantes apliquen el PGTE Para ello, es importante, que, aunque este curso es el primero de los cursos universitarios, se discutan situaciones que son importantes y necesarias de entender como ingenieros y que provoque la curiosidad intelectual, que entiendan que se necesita avanzar en las herramientas y conceptos para seguir adelante y, por consecuencia, comprendan que el PGTE tiene sus limitaciones de aplicabilidad. Sugerimos, entonces, que es necesario investigar y tomar en cuenta los resultados de esta contribución para trabajar sobre el diseño e implementación de materiales didácticos que reduzcan la brecha entre la enseñanza de los conceptos de trabajo y energía, y su comprensión.

\section{Supplementary material}

The following online material is available for this article: Cuestionario

\section{Referencias}

[1] M. Bächtold and M. Guedj, in: International Handbook of Research in History, Philosophy and Science Teaching, edited by M.R. Matthews (Springer Dordrecht, 2014), p. 211-243.

[2] R. Trumper, International Journal of Science Education 15, 139 (1993).

[3] J.L. Domenech, G. Gil-Perez, A. Gras-Marti, J. Guisasola, J. Martinez-Torregrosa, J. Salinas, R. Trumper, P. Valdes and A. Vilches Science \& Education 16, 43 (2007).

[4] B.A. Lindsey, P.R.L. Heron and P.S. Shaffer, American Journal of Physics 77, 999 (2009).

[5] B.A. Lindsey, P.L. Heron and P.S. Shaffer, American Journal of Physics 80, 154 (2012).

[6] J.W. Jewett, The Physics Teacher 46, 38 (2008).

[7] B.A. Sherwood, Am. J. Phys. 51, 597 (1983).

[8] W.H. Bernard, American Journal of Physics 52, 253 (1984).

[9] A.J. Mallinckrodt and H.S. Leff, American Journal of Physics 60, 356 (1992).

[10] K-S Tang, S.C. Tan and J. Yeo International Journal of Science Education 33, 1775 (2011).

[11] M. Cortazzi, Narrative Analysis (Palmer Press, London, 1993).

[12] L. Cohen, L. Manion and K. Morrison, Research Methods in Education (Routledge Taylor and Francis Group, London, 2007).
[13] P. Barniol, E. Campos y G. Zavala in: Physics Education Research Conference Proceedings, Sacramento, edited by D.L. Jones, L. Ding and A. Traxler (PER-Central, Sacramento, 2016) p. 44-47.

[14] T.M. Scaife and A.F. Heckler American Journal of Physics 78, 869 (2010).

[15] P. Barniol and G. Zavala EURASIA Journal of Mathematics, Science and Technology Education 12, 2387 (2016). 